\title{
Altered long- and short-range functional connectivity in patients with long-term unilateral hearing loss
}

\author{
Yanyang Zhang ${ }^{1}$, Zhiqi Mao', Shiyu Feng ${ }^{1}$, Wenxin Wang ${ }^{1,2}$, Jun Zhang ${ }^{1}$ and \\ Xinguang $\mathbf{Y u}^{1}$ \\ ${ }^{1}$ Department of Neurosurgery, Chinese PLA General Hospital, Beijing 100853, China \\ ${ }^{2}$ Department of Radiology, Chinese PLA General Hospital, Beijing 100853, China
}

Correspondence to: Xinguang Yu, email: yuxinguang_301@163.com

Keywords: unilateral hearing loss, resting-state fMRI, functional connectivity, graph theory, neuroscience

Received: April 04, 2017 Accepted: July 30, $2017 \quad$ Published: August 10, 2017

Copyright: Zhang et al. This is an open-access article distributed under the terms of the Creative Commons Attribution License 3.0 (CC BY 3.0), which permits unrestricted use, distribution, and reproduction in any medium, provided the original author and source are credited.

\section{ABSTRACT}

Functionally cross-modal reorganization in the sensory cortex and changes in cognitive processing areas of the brain have been described in patients with bilateral hearing impairments. However, it remains unclear whether long-term unilateral hearing loss (UHL) would similarly influence the higher-order functional networks in addition to the primary sensory networks. In this study, we collected restingstate fMRI data in 21 right-sided UHL patients caused by acoustic neuromas and 21 healthy controls. Whole-brain functional networks were constructed by measuring the temporal correlations of every pairs of brain voxels. We further computed wholerange, short-range and long-range normalized functional connectivity strength (nFCS) for each voxel in the brain. Nonparametric permutation tests were used to detect between-group differences in these nFCS metrics and to further systematically investigate the relationship between these differences and clinical variables in UHL patients. Compared to healthy controls, the UHL patients showed an increased nFCS mainly in anterior cingulate cortex and dorsal medial prefrontal cortex, and these changes resulted from both short-range and long-range nFCS. The UHL patients also showed decreased whole-range nFCS in visual cortex, resulting from short-range functional changes. Additionally, the UHL patients showed decreased long-range nFCS in the orbital frontal cortex. Together, we observed significantly altered nFCS mainly involving in the default-mode, ventral attention, limbic and visual networks. Our study provides empirical evidence for distance-dependent alteration of functional connectivity in long-term UHL, which may implicate the functionally cross-modal reorganization of the brain involving in primary sensory and higher-order functional networks in long-term UHL.

\section{INTRODUCTION}

Studies on congenital or acquired bilateral deafness have observed that individuals deprived of bilateral auditory input can compensate with superior specific abilities in the remaining sensory modalities, including increased tactile sensitivity [1] and improved visual attention to the periphery [2]. In addition, previous research has demonstrated deaf individuals could suffer from different aspects of cognitive dysfunction $[3,4]$. These findings support the hypothesis that the functional changes of brain induced by compromised hearing input are not restricted to the auditory cortex, but also extend across different sensory and higher-order cognitive systems $[5,6]$. Unlike those with bilateral hearing loss, unilateral hearing loss (UHL) individuals preserve much of the ability to capture auditory information [7]. It remains unclear whether this acquired UHL would similarly induce changes of brain in sensory and higher-order areas functionally. Previous studies [3, 4] have demonstrated that in addition to hearing impairments, UHL can contribute to different aspects of brain dysfunctions including cognitive, 
behavioral-emotional, and motor disorders. Moreover, from the connectomic perspective, even the functions of primary sensory areas of the cerebral cortex, once thought to be pinnacles of modularity, are being redefined by recent evidence of cross-modal interactions working together as large-scale networks [8]. Hence, it is theorized that the deprivation of unilateral auditory input in UHL may affect the integral auditory perception, which could propagate further through the widespread brain networks to influence related higher-order psychoacoustic functions. However, until very recently, studies investigated the functional changes associated with UHL were mainly restricted to auditory cortex by examining task-related brain activation. Thus, studies that directly examine the functional changes of brain on the network level in UHL group are warranted.

Resting-state functional magnetic resonance imaging (R-fMRI), which measures ongoing spontaneous brain activity $[9,10]$, has emerged as a powerful tool to map intrinsic typical [11] and atypical [12] brain connectivity networks. Previous functional MRI studies have revealed several UHL-related alterations in specific functional networks, such as the default mode network (DMN) [7, 13], functional networks of auditory [14] and executive control [15], and functional networks of recognition and language [16]. However, these existing studies only focused on specific functional subnetworks rather than integrated communication across the whole brain. The patterns of intrinsic functional connectivity (FC) across the whole brain in UHL patients are still poorly understood. The functional connectivity strength (FCS) $[12,17]$, which provides a voxel-wise index to assess the $\mathrm{FC}$ architecture across the whole brain, could be a sensitive measurement for detecting the functional changes associated with UHL. Specifically, several recent studies have paid more attention to the topology of anatomically embedded brain networks and highlighted the importance of FC distances on brain network organization $[18,19]$. It is claimed that there is an interaction or balance between long- and shortrange $\mathrm{FC}$ in the human brain at resting state to optimize the processing of information $[18,20]$. Importantly, this balance is proved to be changed in specific diseases including obsessive-compulsive disorder [21], epilepsy [22] and minimally treated chronic schizophrenia [23]. However, to our knowledge, there is no study to analyze the alterations of long- and short-range FC and involved functional networks in UHL.

Here, we constructed voxel-wise whole-brain functional connectivity networks by measuring temporal correlations of every pair of brain voxels and further analyzed the normalized FCS (nFCS) in UHL patients caused by right-side acoustic neuroma (AN). Importantly, such a voxel-wise approach avoids parcellation-dependent effects on the topological organization of brain networks $[24,25]$. Additionally, the anatomical distance effect on the nFCS was further studied by dividing the connections into short- and long-range connections according to their anatomical distance [26]. We then evaluate the relationship between regions showing significant between-group nFCS differences and functional Networks. Finally, we assessed the correlation between altered nFCS and the relevant clinical index, such as disease duration or hearing level in UHL patients. We sought to determine (1) whether patients with UHL show alteration of connectivity patterns in their whole-brain functional networks, and (2) if so, whether this alteration is connection-distance-dependent.

\section{RESULTS}

\section{Demographic and clinical data}

There were no significant differences in gender distribution $(p=0.525)$, educational level $(p=0.929)$ and MMSE $(p=0.166)$ between the UHL and healthy control groups. However, significant differences in age $(p=0.010)$ were found. The demographic characteristics and clinical information for both groups are provided in Table 1.

\section{The nFCS maps in UHL patients and healthy controls groups}

The first row of Figure 1 illustrates the wholerange nFCS maps in UHL and healthy controls groups. Visual inspection indicated that the nFCS patterns were remarkably similar between the UHL and healthy control groups in spite of some differences in strength. Those hubs with high nFCS were primarily located in several regions of DMN (mainly involving the precuneus/posterior cingulate cortex, medial prefrontal cortex, and medial, lateral, and inferior parietal cortex), bilateral sensorimotor, and occipital regions (Supplementary Figure 1). This pattern was largely compatible with previous R-fMRI studies in healthy young adults $[27,28]$. Further statistical analysis revealed that, compared to the healthy controls, the UHL patients showed increased nFCS in the left lateral prefrontal cortex (1PFC), dorsal and ventral anterior cingulate cortex (d/vACC), dorsal medial prefrontal cortex (dMPFC) and decreased FCS mainly in visual cortices (Figure 1 and Table 2). Moreover, we found that 38.98\% of the significant voxels demonstrating between-group differences in whole-range nFCS was spatially located in hub regions.

\section{Distance-dependent nFCS alterations in UHL patients}

Row 2 and 3 of Figure 1 show the within- and between-group nFCS maps for short-range nFCS and long-range nFCS, respectively. For healthy group, the regions with highly short-range $\mathrm{nFCS}$ were mainly located in the posterior cingulate cortex/precuneus $(\mathrm{PCC} / \mathrm{PCu})$, $\mathrm{MPFC}$, insula, dACC, sensorimotor, and visual cortices, 
Table 1: Demographics and clinical data of all subjects

\begin{tabular}{lcccc}
\hline & UHL $(\boldsymbol{n}=\mathbf{2 1})$ & Controls $(\boldsymbol{n}=\mathbf{2 1})$ & Statistic (df) & $\boldsymbol{P}$ value \\
\hline Age (years) & 50.19 .6 & 42.88 .0 & $2.688(40)^{\mathrm{a}}$ & 0.010 \\
Gender (Male/Female) & $7 / 14$ & $9 / 12$ & $0.404(1)^{\mathrm{b}}$ & 0.525 \\
Education (years) & 10.83 .6 & 10.83 .2 & $0.089(40)^{\mathrm{a}}$ & 0.929 \\
MMSE & 25.71 .5 & 26.31 .3 & $-1.410(40)^{\mathrm{a}}$ & 0.166 \\
PTA of left ear (dB HL) & 19.32 .6 & 18.72 .2 & $0.876(40)^{\mathrm{a}}$ & 0.386 \\
PTA of right ear (dB HL) & 78.629 .5 & 18.72 .1 & $9.037(40)^{\mathrm{a}}$ & $<0.001$ \\
Duration of UHL (months) & 41.032 .6 & - & & \\
\hline
\end{tabular}

Note: $\mathrm{UHL}=$ unilateral hearing loss; MMSE $=$ Mini-Mental Status Examination; PTA $=$ pure tone average; $\mathrm{HL}=$ hearing level. ${ }^{a} P$-value was obtained using the independent-sample t-test (two-tailed); ${ }^{b} P$-value was obtained using a Pearson Chisquare test (two-tailed).

whereas the regions with highly long-range $\mathrm{nFCS}$ were mainly located in the PCC/PCu, MPFC, IPFC and lateral temporal cortices. Notably, visual examination indicated that the patterns of both short- and long-range nFCS maps in the UHL group exhibited similar to those in the healthy controls group. Further group comparisons revealed significantly higher short-range $\mathrm{nFCS}$ values in
UHL group in left IPFC, ACC and dMPFC. Additionally, the UHL patients displayed lower short-range nFCS in the visual cortices (Row 2 of Figure 1 and Table 2). Compared to the healthy controls, the UHL patients showed decreased long-range $\mathrm{nFCS}$ in the orbital frontal cortex and increased long-range $\mathrm{nFCS}$ in the $\mathrm{dACC}$ and dMPFC (Row 3 of Figure 1 and Table 2).

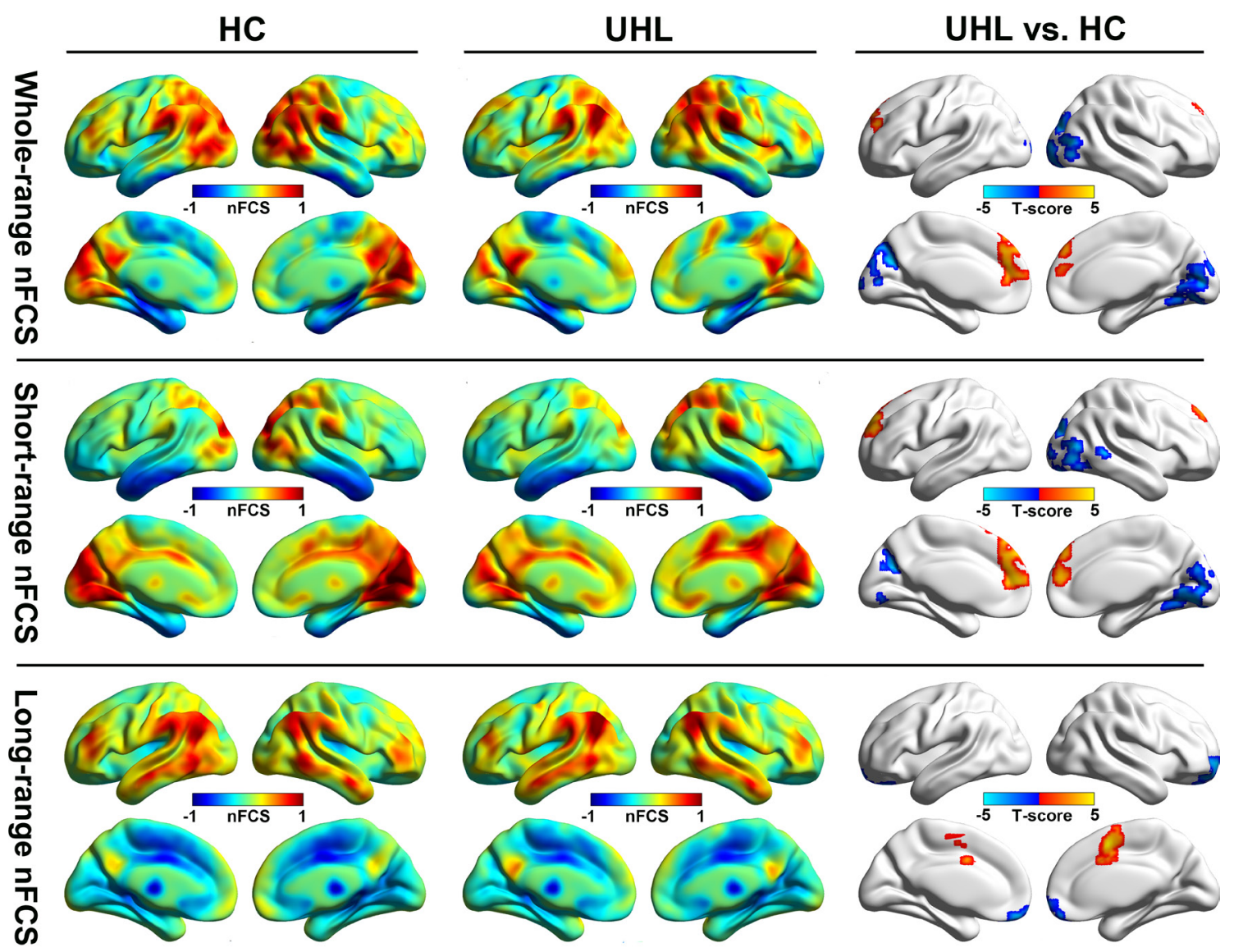

Figure 1: The nFCS maps. The first column and second column show the mean nFCS maps within HC and UHL groups. The last column illustrates the difference of nFCS between the two groups in those connectivity metrics (whole-range nFCS, short-range $n F C S$ and long-range $\mathrm{nFCS})$ respectively, represented as a T score $(p<0.05$, FWE corrected). HC, healthy control; UHL, unilateral hearing loss; nFCS, normalized functional connectivity strength. 


\section{Results in relation to functional networks}

Based on the 7-network parcellation (Figure 2D) described by Yeo et al. [29], the number of significant voxels spatially located in each of the seven networks was determined, expressing as a percentage of the total number of significant voxels (Figure 2E). Regions showing altered whole-range $\mathrm{nFCS}$ mainly belonged to $\mathrm{DMN}(36.9 \%)$ and visual network (59.4\%) (Figure 2A). Meanwhile, UHL patients also demonstrated altered short-range $\mathrm{nFCS}$ in DMN (51.9\%) and visual network (42.7\%) (Figure 2B). Additionally, we found that the altered long-range nFCS regions prevailingly located in higher-order networks including DMN (21.5\%), ventral attention network $(32.7 \%)$ and limbic network (30.9\%) (Figure 2C).

\section{Relationship between nFCS and clinical variables in UHL patients}

To note, within the clusters demonstrating betweengroup differences, we did not find significant correlations between those connectivity metrics (whole-range $\mathrm{nFCS}$, short-range $\mathrm{nFCS}$ and long-range $\mathrm{nFCS}$ ) with the clinical variables including hearing loss duration or hearing level.

\section{Reproducibility of our findings}

As shown in Figure 3, the correlation coefficient thresholds (Figure 3A and Figure 3B), whole brain signal removal (Figure 3C), head motion 'scrubbing' (Figure 3D) and un-normalized FCS maps (Figure 3E) showed minimal
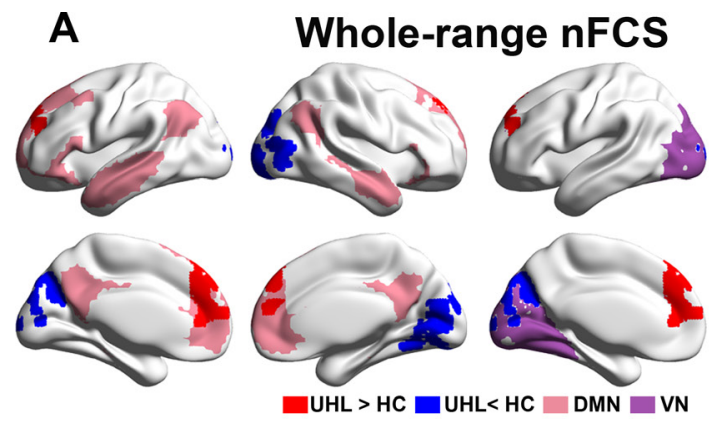

B

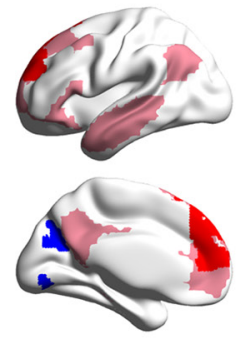

C

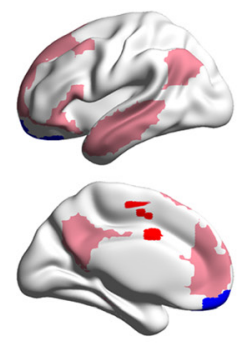

Short-range nFCS

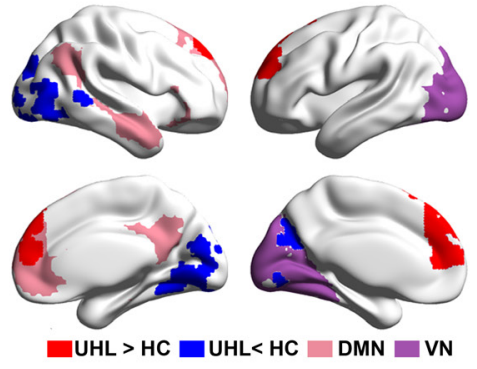

\section{Long-range nFCS}
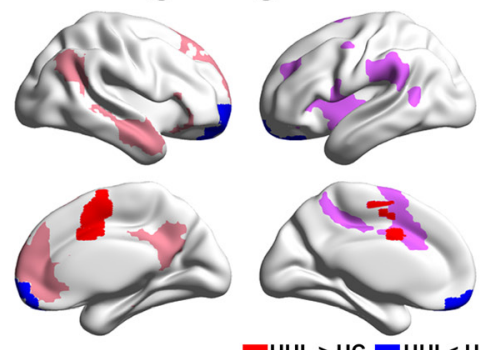
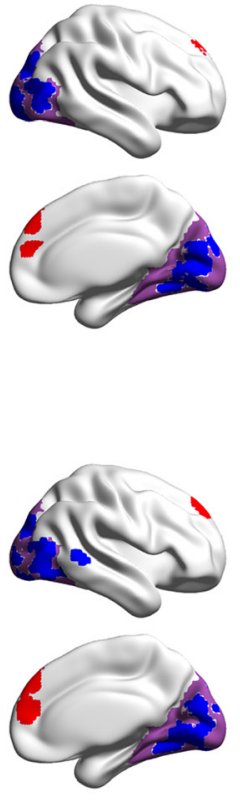

D 7-Network Parcellation
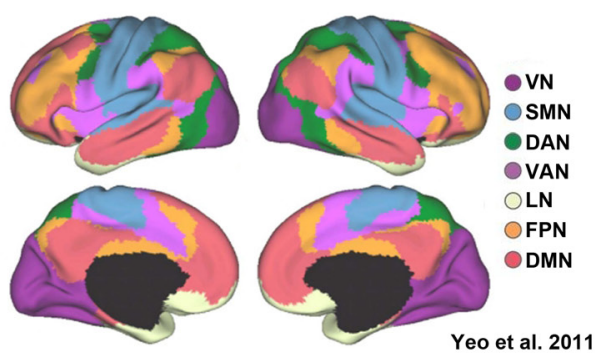

E Fingerprint plots of $\mathrm{nFCS}$

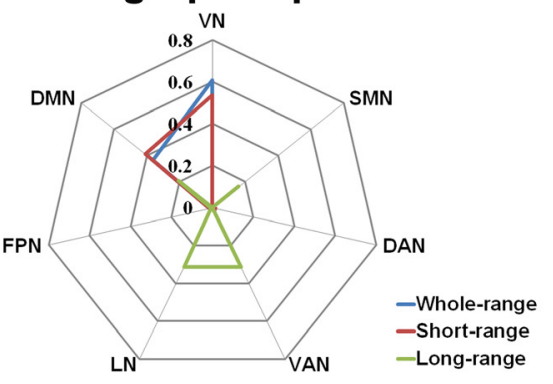

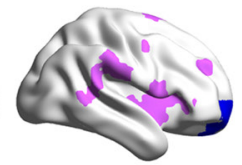
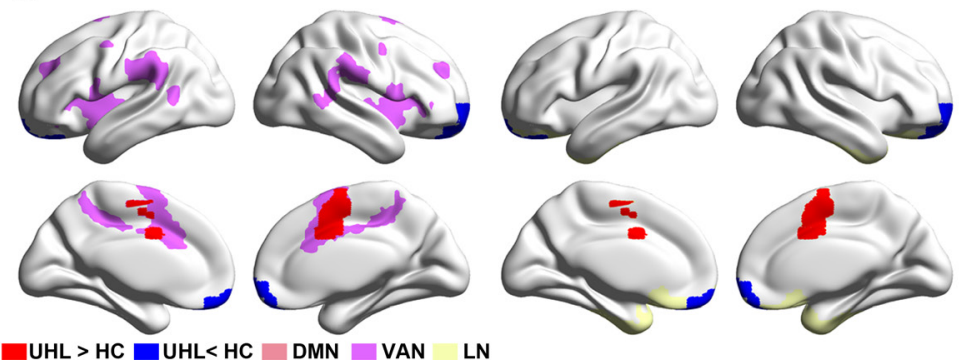

$\square$ UHL > HC $\square$ UHL $<$ HC $\square$ DMN $\square$ VAN LN

Figure 2: Relationship between regions showing significant between-group nFCS differences and functional Networks. (A-C) demonstrated the spatial distribution of the overlap between the regions showing significant between-group differences and the prevailingly involved functional networks in whole-range nFCS, short-range nFCS and long-range nFCS respectively, based on the 7-network parcellation (D) described by Yeo [29]. (E) fingerprint plots showed regions with altered whole-range nFCS and short-range $\mathrm{nFCS}$ were mainly in $\mathrm{VN}$ and DMN, while those altered long-range $\mathrm{nFCS}$ regions mainly located in higher-order networks including DMN, VAN and LN. HC, healthy control; UHL, unilateral hearing loss; nFCS, normalized functional connectivity strength; VN, visual network; SMN, somatomotor network; DAN, dorsal attention network; VAN, ventral attention network; LN, limbic network; FPN, frontoparietal network; DMN, default mode network. 


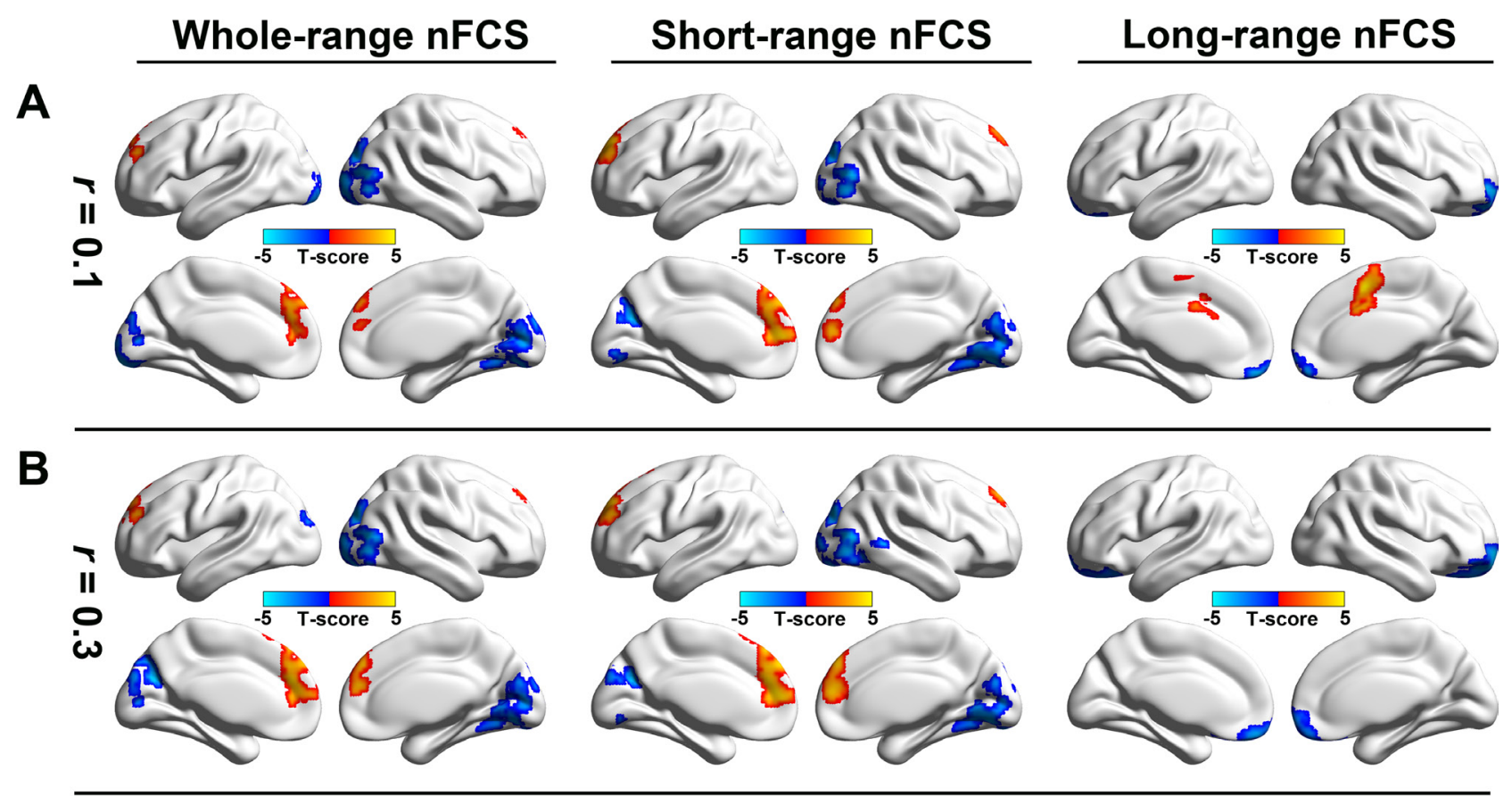

C
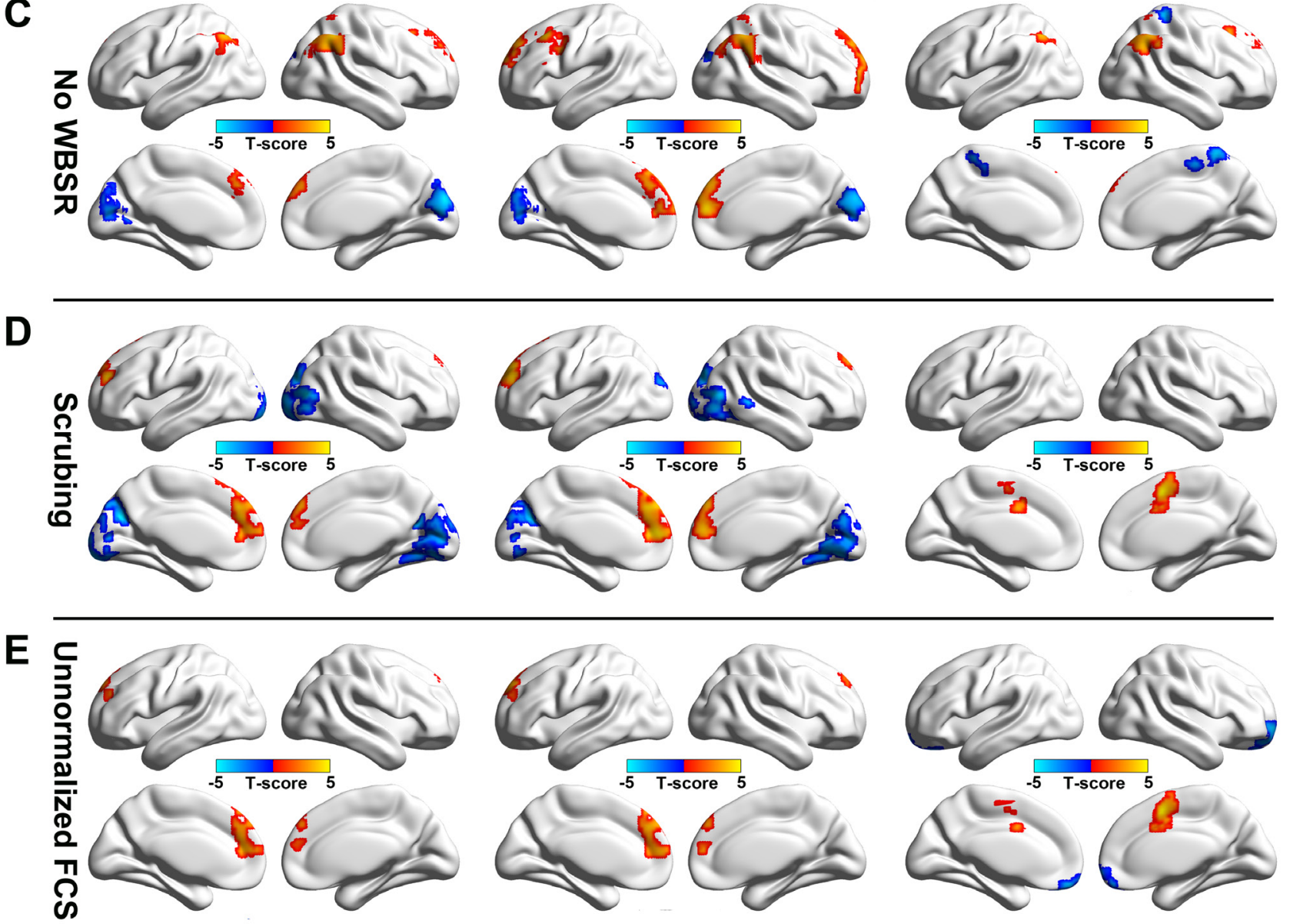

Figure 3: Validation of the findings. Between-group differences in whole-range nFCS, short-range nFCS and long-range $n F C S$ were calculated using correlation threshold of 0.1 (A) and $0.3(\mathbf{B})$; without global signal removal $(\mathbf{C})$ and head motion 'scrubbing' procedure (D), respectively. Figure (E) demonstrated the differences of un-normalized FCS between the two groups. nFCS, normalized functional connectivity strength; WBSR, whole brain signal regression. 
Table 2: Brain regions showing different nFCS between HCs and UHL patients

\begin{tabular}{|c|c|c|c|c|c|c|c|}
\hline & & \multirow[t]{2}{*}{ Brain regions } & \multirow{2}{*}{$\begin{array}{c}\text { Cluster size } \\
\text { (voxels) }\end{array}$} & \multicolumn{3}{|c|}{ Peak MNI coordinate } & \multirow[t]{2}{*}{ Peak } \\
\hline & & & & $\mathbf{x}$ & $\mathbf{y}$ & $\mathbf{z}$ & \\
\hline \multirow[t]{2}{*}{ Whole-brain nFCS } & $\mathrm{HC}>\mathrm{UHL}$ & Bilateral Calcarine sulcus & 674 & 42 & -75 & 9 & -4.63 \\
\hline & $\mathrm{HC}<\mathrm{UHL}$ & Bilateral medial superior frontal gyrus & 491 & 0 & 48 & 51 & 5.49 \\
\hline \multirow[t]{2}{*}{ Short-range nFCS } & $\mathrm{HC}>\mathrm{UHL}$ & Bilateral lingual gyrus & 793 & 42 & -72 & 6 & -5.39 \\
\hline & $\mathrm{HC}<\mathrm{UHL}$ & Bilateral medial superior frontal gyrus & 836 & -9 & 42 & 33 & 5.31 \\
\hline \multirow[t]{2}{*}{ Long-range $\mathrm{nFCS}$} & $\mathrm{HC}>\mathrm{UHL}$ & Bilateral orbital superior frontal gyrus & 264 & 21 & 63 & -6 & -4.09 \\
\hline & $\mathrm{HC}<\mathrm{UHL}$ & Left medial superior frontal gyrus & 232 & 6 & 6 & 51 & 5.08 \\
\hline
\end{tabular}

Note: $\mathrm{nFCS}=$ normalized functional connectivity strength; $\mathrm{HC}=$ healthy control; $\mathrm{UHL}=$ unilateral hearing loss.

effects on group differences compared with our main findings. Moreover, before using a z-score to compare these groups in nFCS maps, we found no systematic difference between groups in mean with-group FCS (Supplementary Table 1). It was worth mentioning that in the analysis with un-normalized FCS maps, the differences of FCS in visual cortices between the UHL patients and controls were not found, which could be attributed to inter-individual variations. Additionally, without global signal regression, we found more widespread between-group differences in long-range nFCS. Finally, for the SNR of the fMRI signals in the orbital frontal cortices, We observed that the SNR of fMRI signals in patients group is on average 66.26 and ranges between 27.23 and 146.08, For the fMRI data in controls group, the mean SNR is 56.84, ranging from 20.30 to 155.70 . Based on these results, we found that the data quality of the resting-state data in patients group is highly identical to that in controls group $(P=0.405)$.

\section{DISCUSSION}

In this exploratory study we investigated voxelwise whole-brain functional connectivity patterns in UHL patients. We found that, although both UHL patients and healthy controls groups showed similar patterns of wholerange, short-range and long-range $\mathrm{nFCS}$, further statistical analysis revealed significant connectivity abnormalities in UHL group and these abnormalities were distancedependent. Specifically, we observed significantly increased short-range nFCS in the left $1 \mathrm{PFC}, \mathrm{ACC}$, dMPFC and decreased in the visual cortices. Additionally, we also observed increased long-range nFCS in the $\mathrm{dMPFC}, \mathrm{dACC}$ and decreased nFCS in the orbital frontal cortex. These regions with altered nFCS were found involved in multimodal networks including DMN, ventral attention, limbic and visual network. Our findings suggest that deprivation of unilateral auditory input could affect the neural nFCS of higher-order functional network in addition to sensory cortex and this pattern of altered nFCS is distance-dependent.

\section{Altered whole-range nFCS in UHL}

Compared to healthy controls group the UHL patients showed significantly increased nFCS in the left
dlPFC, d/v ACC, dMPFC. Previous studies [7, 13, 30] indicated that unilateral sensory input damage not only alters the activity of the sensory areas but also reshapes the regional and circuit functional organization of the cognitive network, which was compatible with our findings. Extensive, increased nFCS in several areas of the DMN, especially in the dMPFC, left dlPFC and ACC in UHL patients, are prominent findings in this study. In line with our study, previous R-fMRI studies [7] have reported UHL patients presented increased functional connectivity in the cognitive control network and DMN. Furthermore, Zhang et al. [13] found that patients with UHL demonstrated enhanced connectivity and significant changes of the nodal topological properties in the DMN compared with the healthy controls. The dlPFC is been found to be associated with a variety of cognitive functions, such as the maintenance and manipulation of information in working memory, intention formation, goaldirected action, abstract reasoning, decision-making, and attentional control [31]. Recent studies [32] also suggest that dlPFC-mediated cognitive control functions may also pertain to emotion. Specifically, functional imaging studies $[33,34]$ demonstrate the dlPFC is recruited during the regulation of negative emotion through reappraisal/ suppression strategies. The dMPFC is involved in aspects of cognitive control and emotional regulation as well [35, 36]. Various findings [28, 37] demonstrated that ACC is a part of a circuit involved in a form of attention that serves to regulate both cognitive and emotional processing. The changes of nFCS in these regions, identified by us in the study, suggest that UHL patients are associated with alternations in a neural system that subserves cognitive and emotional control functions, and which may be related to cognitive bias. This is in accordance with the evidence that the internal connectivity within sensory and higher-order cognitive networks might functionally be reorganized in UHL patients.

Further, we found enhanced nFCS in several areas of DMN in UHL compared to control subjects, suggesting reorganization within the DMN. In healthy subjects, it is recognized that the DMN [38] is involved in cognitive processes - for example, emotional processing and selfreferential mental activity [39], conflict monitoring 
[37], memory retrieval [40] and cognitive control [41]. Numerous studies have found that the DMN can be affected by neurological and psychiatric diseases [42, 43]. In the study [13], worse performance in most of behavior tests was found in UHL than the controls, however, significant difference was not reached. Similarly, no significant differences were found in MMSE in our study. One possible reason is that UHL-related functional reorganization increases cognitive processing abilities to limit the consequences of neurological damage and to help maintain cognitive abilities [44]. Such functional reorganization is manifested as enhanced functional connectivity in several regions in the DMN. Therefore, the enhanced connectivity in the DMN of the UHL might be a compensation for the decline of cognition.

Another major finding in this study was reduced $\mathrm{nFCS}$ in visual cortices in UHL patients relative to that in healthy controls, which may present the baseline abnormal nFCS of sensory cortices in UHL. In line with our study, previous structural imaging studies [30, 45] have shown decreased gray matter volume in lingual gyrus in the patients with right-sided hearing loss. Furthermore, using fMRI, Schmithorst et al. [46] found significantly less functional activation in visual processing regions while performing a classic receptive language test in children with right or severe-to-profound UHL. Of note, Wang et al. [7] also found decreased regional homogeneity $(\mathrm{ReHo})$ in the bilateral calcarine cortices in UHL. In fact, direct connections from primary and secondary auditory areas, into primary and secondary visual areas, have been shown in monkeys via tracer studies $[47,48]$. Thus, partial sensory deprivation of one modality (auditory) could affect the functions of the remaining modalities (visual) $[7,46]$. Our results suggest that reorganization of visual processing is affected by the sensory deprivation stemming from the progressive UHL caused by AN. It should also be acknowledged, however, other previous research of auditory brain plasticity on congenitally deaf individuals have reported that bilateral deafness is associated with activation and hyper-functionality in the visual cortex when having auditory stimulation $[49,50]$. It is possible that UHL patients largely preserve the ability to capture auditory information in the unaffected ear, which likely ameliorates downstream effects on visual processes. Of note, after considering the effects of anatomical distance, UHL only caused local short-range functional changes in visual areas.

It is worth noting that, the auditory cortex is expected to be functionally reorganized in UHL patients intuitively. However, in current study, UHL patients did not exhibit significantly changed nFCS involving in auditory areas. Such negative findings may be due to the fact that all UHL patients in current study were postlingual deaf. Previous study revealed that the age of the onset of deafness was critical to cortical plasticity $[7,51]$. Hence, we inferred that the plastic changes within auditory cortex of acquired UHL patients might be too mild to be significant statistically. Additionally, we found the changes of nFCS in both hemispheres. In normal hearing, the auditory information from each ear is processed by both ipsilateral and contralateral auditory cortices. It follows that the dysfunction of auditory input in right ear interrupts both hemispheres. To draw a more generalized conclusion, the lateralization of effects in the brain warrants further studies including left-sided HL patients.

\section{The significance of changed short- and long- range $\mathrm{nFCSs}$ for $\mathrm{UHL}$}

In healthy populations, there is a balance between local (short-range) area interactions and distant (longrange) projections which could optimize the brain information processing to make it highly efficient and low-costing [18]. Short- and long-range connectivity represent for projections from local and remote brain areas, respectively. This separation might deepen our understanding about the information processing mixed with projections from both the adjacent and distant brain systems. In our study, we found changed long- and shortrange $\mathrm{nFCS}$ in USL patients compared with healthy controls, mainly involving DMN, the ventral attention and visual network. These facts might implicate a functional deficit basis of UHL patients to some extent.

Regions with abundant short-range connectivity, are mainly located in the primary sensory and motor cortical areas, and are recognized as short-range connection hubs [52]. In contrast, hetero-model cortical areas have abundant long- range connections, and that the core components of the DMN as well as limbic regions have both abundant short- and long- range connectivity [20]. In our study, we found an overlap of higher long- and shortrange FCS mainly located in brain regions comprising DMN, which highlights the functional changes of higherorder networks in processing both local and distant information of brain in UHL patients. One possible reason is that such enhanced nFCS occurs to compensate for the impairment of cognition induced by long-term hearing loss. However, on the other hand, such abnormal increase of nFCS in DMN, which may have the ability to improve information processing in theory, could be counterproductive because information is being sent to the wrong brain regions. In addition, in visual network, significantly decreased short-range $\mathrm{nFCS}$ was found in UHL patients. These findings together support the notion that adaptive sensory drives multi-modal plasticity involved in the sensory and higher-order networks.

Greater long-range nFCS indicates a shorter path length such that the efficiency of information management and processing is more efficient [53] and vice versa. In our study, we found a reduction of long-range $\mathrm{nFCS}$ in orbital frontal gyrus among UHL patients. The orbital frontal gyrus, one cortical component of temporal-amygdala- 
orbitofrontal network (connected through the uncinate fasciculus) in the limbic system, serves to suppress negative affect by inhibiting amygdalar output to maintain affective states [54]. Abnormal activity in the orbital frontal cortex is widely reported in the studies of mood and anxiety disorders $[54,55]$. Thus, it is conceivable that a reduction of long-range $\mathrm{nFCS}$ in orbital frontal cortex might influence emotional states with cognition and behavior in UHL patients. This result is consistent with previous studies suggesting acquired UHL is associated with language, speech, and behavioral deficits.

\section{Limitations and future works}

There are several methodology issues and limitations in this study. First, in the context of fMRI research, a technological issue is the possible confounding effect of acoustic scanner noise (ASN) on intrinsic connectivity networks [56]. Moreover, the ASN is perceived at a lower level or may be inaudible for the affected ear in the UHL patients. Thus, the changes of nFCS might be attributable to the effect of both long-term UHL and ASN on the brain. To circumvent concerns about ASN, further refined studies, for instance by not plugging the affected ear during the fMRI scan, are warranted. Second, we analyzed the resting-state nFCS using a cross-sectional design in chronic UHL. It should be acknowledged that such a design limited our ability to discriminate what is a consequence of chronicity, or progression and what is a stable pattern in UHL. A future longitudinal followup study could help to clarify this issue. Moreover, the patients with UHL were all caused by right AN, although we assumed that this subtentorial tumor could not affect hemispheric plasticity in UHL, further studies including data on UHL caused by other etiologies would provide more specific information. Third, this study only examined whole-brain nFCS changes in patients with right-sided HL. It would be of great interest to ascertain whether similar alterations of $\mathrm{nFCS}$ could be observed in patients with left-sided HL, which could deepen our understanding of the influences of UHL on brain. Furthermore, the structural foundation underlying these UHL-related functional changes needs to be explored by a combined analysis of multimodal imaging data. Finally, further works combining extensive cognitive profiling and nFCS analysis are warranted to uncover the neuronal circuitries underlying specific cognitive deficits in UHL.

\section{MATERIALS AND METHODS}

\section{Patients}

This study is comprised of 42 subjects: $21 \mathrm{UHL}$ patients with primary untreated right-side $\mathrm{AN}$ and 21 healthy controls. Pure tone audiometry with six different octave frequencies $(0.25,0.5,1,2,4$ and $8 \mathrm{kHz})$ was performed by a clinical audiometer to measure the pure tone average (PTA) and reflect hearing level. All patients in this study were diagnosed with right-side hearing loss with hearing deficit in the right ear (PTA $\geq 40 \mathrm{~dB} H \mathrm{HL})$ and normal hearing in the left ear (PTA $\leq 25 \mathrm{~dB}$ HL). The control subjects with PTA below $25 \mathrm{~dB}$ for both ears were well matched to the patient group in terms of gender and education level, except for age (Table 1). The cognition of all participants was evaluated by the Mini-Mental State Examination (MMSE) [57], which has been widely used clinically for screening cognitive impairment. Individuals were excluded if they had a history of medical, psychiatric disorders, strokes/cerebrovascular ischemia, head trauma/ fractures. All participants were right-handed which was determined using the Edinburgh Handedness Scale. The study was approved by the local ethics committee of the Chinese People's Liberation Army (PLA) General Hospital and written informed consent was obtained from each participant.

\section{Image acquisition}

MRI data acquisition was performed on a GE750 3.0 T scanner in the Department of Radiology of PLA General Hospital. Participants were fitted with soft earplugs and positioned carefully in the coil with comfortable support. The functional images were obtained using an echo-planar imaging sequence with the following parameters: repetition time $=2000 \mathrm{~ms}$, echo time $=30 \mathrm{~ms}$, flip angle $=90^{\circ}$, thickness $/$ gap $=3.5 \mathrm{~mm} / 0.5 \mathrm{~mm}$, slices $=36$, field of view $=224 \times 224 \mathrm{~mm}^{2}$ and voxel size $=3.5 \times 3.5 \times 3.5 \mathrm{~mm}^{3}$. High-resolution structural images were collected using a sagittal Fast Spoiled Gradient-Echo (FSPGR) sequence with the following parameters: repetition time $=6.7 \mathrm{~ms}$, echo time $=2.9 \mathrm{~ms}$, flip angle $=7^{\circ}$, thickness $=1 \mathrm{~mm}$, slices $=192$, field of view $=256 \times 256 \mathrm{~mm}^{2}$ and voxel size $=1 \times 1 \times 1 \mathrm{~mm}^{3}$. During the scan, participants were instructed to lie still with their eyes closed and to avoid falling asleep or think of anything in particular during the data acquisition. After the scanning, a simple questionnaire indicated that no participants had fallen asleep.

\section{Data preprocessing}

Image preprocessing was carried out using Statistical Parametric Mapping (SPM12, http://www.fil.ion.ucl. ac.uk/spm) and Data Processing Assistant for RestingState fMRI (DPARSF) [58]. For the signal equilibrium and participants' adapting to the circumstances, the first ten volumes were discarded. The remaining functional scans were first corrected for within-scan acquisition time differences between slices and further realigned to the first volume to correct for inter-scan head motions. For head motion, all subjects in this study had no more than $3 \mathrm{~mm}$ translation in the $\mathrm{x}, \mathrm{y}$, or $\mathrm{z}$-axes and less than 3 degree of rotation in each axis. Next, the individual T1-weighted 
images were coregistered to the mean functional image after motion correction using a linear transformation and were then segmented into gray matter (GM), white matter, and cerebrospinal fluid tissue maps with SPM's a priori tissue maps as reference by using a unified segmentation algorithm [59]. We then applied the transformation parameters estimated during unified segmentation to the motion-corrected functional volumes and resampled the transformational functional images to 3-mm isotropic voxels. Subsequently, we regressed out the nuisances, including the global signal, the white matter signal, the cerebrospinal fluid signal, and 24 head motion parameters (6 motion parameters for the current the volume, 6 motion parameters for the previous volume and 12 corresponding squared items) estimated in the image realignment step. Finally linear de-trending and temporal band-pass filtering $(0.01-0.08 \mathrm{~Hz})$ were performed to reduce the effects of low-frequency drift and high-frequency physiological noise.

\section{Whole-brain functional connectivity strength analysis}

The analysis workflow is illustrated in Figure 4. To perform a voxel-wise whole-brain functional connectivity analysis on the preprocessed R-fMRI data, we used a datadriven method which has been used in several previous studies [12, 43, 52, 60]. Briefly, for each participant, Pearson's correlations were computed between the time series of all pairs of brain voxels and a whole-brain FC matrix for each participant was obtained. The computation was constrained within a gray-matter mask $\left(\mathrm{N}_{\text {voxels }}=\right.$ 45381) which was generated by thresholding (a threshold of 0.2 ) a prior gray-matter probability map in SPM8. To eliminate the voxels with weak correlations possibly arising from signal noises, prior to calculating FCS, our cross-correlation analysis was further conservatively restricted to positive correlations above a correlation coefficient threshold of $r 0\left(r_{0}=0.2\right.$ in this study). Given a gray-matter voxel $i$, its FCS is computed using the following equation:

$$
F C S(i)=\frac{1}{N-1} \sum_{j \neq i} Z_{i j}, r_{i j}>r_{0}
$$

where $N$ denoted the total number of voxels in the gray matter mask, $r_{\mathrm{ij}}$ was the correlation coefficient between voxel $i$ and voxel $j, r_{0}$ was the correlation coefficient threshold ( $r_{0}=0.2$ in this study), the $r_{\mathrm{ij}}$ was converted to $z_{\mathrm{ij}}$ using Fisher $Z$-transformation to improve normality. Of note, FCS, referred to as the "degree centrality" of weighted networks, has been widely used to examine the characteristics of nodes in functional networks $[12,61]$ and has a demonstrated close relationship with cerebral blood flow [52] and oxidative glucose metabolism [62].

To minimize the influences of nuisance variables on inter-individual variation in FCS and make the FCS as a comparable scale between subjects, we further computed the normalized FCS for a given voxel $i$ using the following equation:

$$
n F C S(i)=\frac{F C S(i)-\overline{F C S}}{\sigma(F C S)}
$$

Where $\overline{F C S}$ is the mean FCS across all the voxels in the GM mask, and $\sigma(F C S)$ is the standard deviation of the FCS in the GM mask. The GM voxels with higher nFCS values ( $>1$ SD beyond the global mean) were defined as brain hubs [43].

To explore the effects of anatomical distance on connectivity analysis, we further divided whole-range nFCS into two categories, short-range nFCS and long-
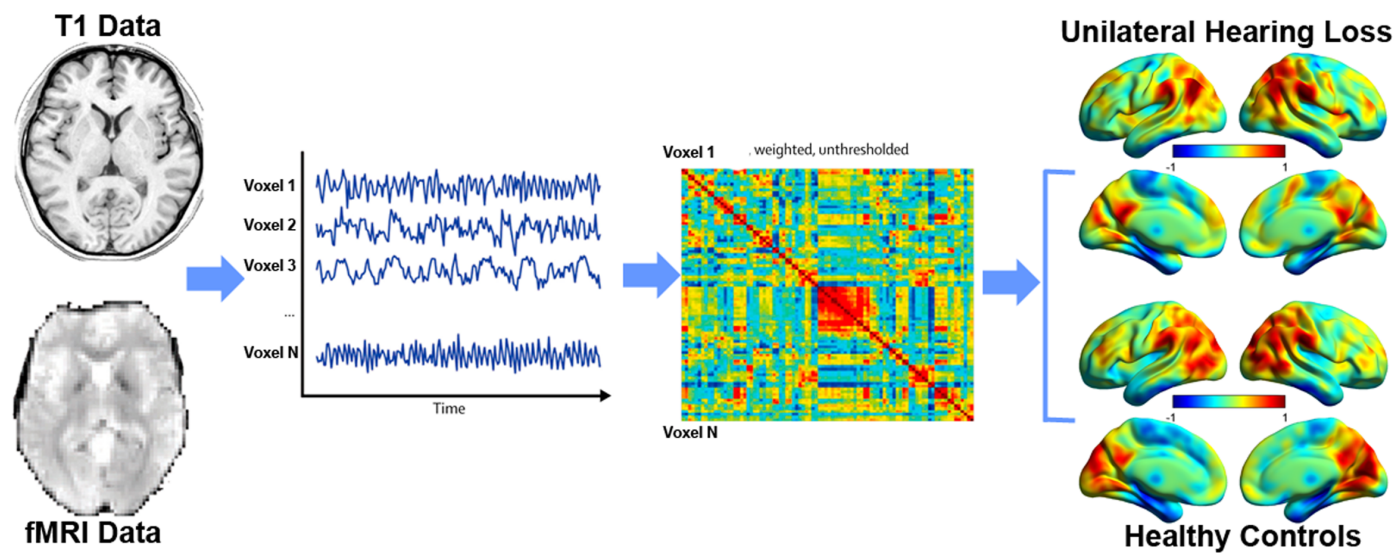

Figure 4: Analysis workflow in this study. The time series for each voxel was extracted from the preprocessed resting-state fMRI data to calculate a correlation matrix. We then constructed voxel-level weighted graphs, where nodes represented brain voxels and edges represented inter-voxel functional connectivity. Significant between-group differences in functional connectivity strength were further identified. 
range nFCS [18]. The short-range FCS of a voxel referred to the sum of those connections (Z-values) between the voxel and other GM voxels with anatomical distances less than $75 \mathrm{~mm}$ to the given voxel, whereas the long-range FCS of a voxel referred to the sum of its connections (Z-values) with distances greater than $75 \mathrm{~mm}$ [26]. In this study, the anatomical distance between two GM voxels was defined as the Euclidean distance (approximately corresponding to anatomical distances) between their MNI coordinates. Finally, all individual nFCS maps were spatially smoothed with a Gaussian kernel (full width at half-maximum $=6 \mathrm{~mm}$ ).

\section{Altered nFCS in relation to functional networks}

We further spatially related the voxels demonstrating between-group differences in those connectivity metrics (whole-range nFCS, short-range $\mathrm{nFCS}$ and long-range nFCS) to seven reference networks defined by Yeo et al. [29] on the basis of a data-driven analysis of resting state functional imaging data collected from 1,000 participants. We determined the involved networks by computing the number of significant voxels from the above comparison that spatially overlapped each of the network masks (downloaded from http://surfer.nmr.mgh.harvard.edu/ fswiki/ CorticalParcellation_Yeo2011).

\section{Statistical analysis}

Age, years of education, the scores of MMSE and hearing levels were compared by two-sample $t$-tests. Sex composition of the two groups was compared using a Pearson Chi-square test (two-tailed). To explore differences in whole-range nFCS, short-range nFCS and long-range nFCS between UHL patients and healthy controls, we performed nonparametric permutation tests based on 10000 permutations for each nFCS metric. This procedure was conducted by using the Statistical nonParametric Mapping (SnPM) toolbox (http://www. nitrc.org/projects/snpm/) in SPM12. SnPM uses the General Linear Model to construct pseudo t-statistic images, which are then assessed for significance using a standard non-parametric multiple comparisons procedure testing [63]. We note that a recent study [64] has reported the reliability of nonparametric permutation method in controlling false positive rate in the cluster-level inference. In this study, we set significance level as the cluster-forming threshold of 0.01 with familywise error rate (FWE) corrected cluster $p<0.05$.

Then, to investigate the relationship between nFCS and clinical variables including disease duration or hearing level in UHL group, a voxel-based multiple linear regression analysis was separately conducted in those connectivity metrics (whole-range nFCS, shortrange $\mathrm{nFCS}$ and long-range $\mathrm{nFCS}$ ) within the clusters demonstrating between-group differences, considering age, gender and education level as covariance. Multiple comparisons were corrected again using nonparametric permutation ( $p<0.05$, FWE corrected).

\section{Validation analysis}

To validate our nFCS results, we adopted several procedures to assess the effect of different analytic strategies. (1) The effect of correlation coefficient threshold; as mentioned above, we used a single correlation coefficient threshold of 0.2 to eliminate weak correlations possibly arising from signal noise while computing nFCS. To determine whether the main results were dependent on the correlation thresholds, additional nFCS maps were calculated using other two different correlation thresholds (i.e., 0.1 and 0.3). (2) The effect of whole brain signal regression (WBSR); we additionally investigated whether or not without regressing out the global signal during nFCS calculations would affect the findings. (3) The effect of head motion; we re-performed a 'scrubbing' procedure on the preprocessed images [65]. R-fMRI volumes were first censored based on a criterion of framewise displacement $>$ $0.5 \mathrm{~mm}$, and the nFCS maps were then re-analyzed using these censored R-fMRI data. (4) The effect of un-normalized FCS maps; in current study, the FCS map was further normalized by converting to $Z$ scores so that maps across participants could be averaged and compared. To evaluate the reproducibility of our results, the un-normalized FCS maps were also calculated. (5) The effect of susceptibility artifacts; in current study, we calculated the signal-tonoise ratio (SNR) of the fMRI signals in the orbital frontal cortices. The SNR is the quotient of the (mean) signal intensity measured in a region of interest (the orbital frontal cortices) and the standard deviation of the signal intensity in a region from which no tissue signal is obtained [66].

\section{Author contributions}

Yanyang Zhang, acquisition and interpretation of the data, drafting the manuscript. Zhiqi Mao, study concept and design, acquisition of data, study supervision. Shiyu Feng, revising the manuscript for content. Wenxin Wang, acquisition of data, revising the manuscript for content. Jun Zhang, revising the manuscript for content. Xinguang $\mathrm{Yu}$, study concept and design, acquisition of data, make the diagnosis and study supervision.

\section{CONFLICTS OF INTEREST}

The authors report no declarations of interest.

\section{REFERENCES}

1. Levänen S, Hamdorf D. Feeling vibrations: enhanced tactile sensitivity in congenitally deaf humans. Neurosci Lett. 2001; 301:75-77. 
2. Bavelier D, Tomann A, Hutton C, Mitchell T, Corina D, Liu G, Neville H. Visual attention to the periphery is enhanced in congenitally deaf individuals. J Neurosci. 2000; 20:RC93.

3. Calderón-Leyva I, Díaz-Leines S, Arch-Tirado E, LinoGonzález AL. Analysis of the relationship between cognitive skills and unilateral sensory hearing loss. Neurologia. 2016.

4. Chilosi AM, Comparini A, Scusa MF, et al. Neurodevelopmental disorders in children with severe to profound sensorineural hearing loss: a clinical study. Dev Med Child Neurol. 2010; 52:856-62.

5. Bavelier D, Neville HJ. Cross-modal plasticity: where and how. Nat Rev Neurosci. 2002; 3:443-452.

6. Merabet LB, Pascual-Leone A. Neural reorganization following sensory loss: the opportunity of change. Nat Rev Neurosci. 2010; 11:44-52.

7. Wang X, Fan Y, Zhao F, et al. Altered regional and circuit resting-state activity associated with unilateral hearing loss. PLoS One. 2014; 9:e96126.

8. Menon V. Large-scale brain networks and psychopathology: a unifying triple network model. Trends Cogn. Sci. (Regul. Ed.). 2011; 15:483-506.

9. Biswal B, Yetkin FZ, Haughton VM, Hyde JS. Functional connectivity in the motor cortex of resting human brain using echo-planar MRI. Magn Reson Med. 1995; 34:537-41.

10. Fox MD, Raichle ME. Spontaneous fluctuations in brain activity observed with functional magnetic resonance imaging. Nat Rev Neurosci. 2007. 8:700-11.

11. He Y, Wang J, Wang L, et al. Uncovering intrinsic modular organization of spontaneous brain activity in humans. PLoS One. 2009; 4:e5226.

12. Buckner RL, Sepulcre J, Talukdar T, et al. Cortical hubs revealed by intrinsic functional connectivity: mapping, assessment of stability, and relation to Alzheimer's disease. J Neurosci. 2009. 29:1860-73.

13. Zhang GY, Yang M, Liu B, Huang ZC, Chen H, Zhang PP, Li J, Chen JY, Liu LJ, Wang J, Teng GJ. Changes in the default mode networks of individuals with long-term unilateral sensorineural hearing loss. Neuroscience. 2015; 285:333-342.

14. Suzuki M, Kouzaki H, Nishida Y, Shiino A, Ito R, Kitano H. Cortical representation of hearing restoration in patients with sudden deafness. Neuroreport. 2002; 13:1829-1832.

15. Tibbetts K, Ead B, Umansky A, Coalson R, Schlaggar BL, Firszt JB, Lieu JE. Interregional brain interactions in children with unilateral hearing loss. Otolaryngol Head Neck Surg. 2011; 144:602-611.

16. Liu B, Feng Y, Yang M, Chen JY, Li J, Huang ZC, Zhang LL. Functional Connectivity in Patients With Sensorineural Hearing Loss Using Resting-State MRI. Am J Audiol. 2015; 24:145-152.

17. Bassett DS, Bullmore ET, Meyer-Lindenberg A, Apud JA, Weinberger DR, Coppola R. Cognitive fitness of cost- efficient brain functional networks. Proc Natl Acad Sci USA. 2009; 106:11747-11752.

18. Alexander-Bloch AF, Vértes PE, Stidd R, Lalonde F, Clasen L, Rapoport J, Giedd J, Bullmore ET, Gogtay N. The anatomical distance of functional connections predicts brain network topology in health and schizophrenia. Cereb Cortex. 2013; 23:127-138.

19. Vértes PE, Alexander-Bloch AF, Gogtay N, Giedd JN, Rapoport JL, Bullmore ET. Simple models of human brain functional networks. Proc Natl Acad Sci USA. 2012; 109:5868-5873.

20. Sepulcre J, Liu H, Talukdar T, Martincorena I, Yeo BT, Buckner RL. The organization of local and distant functional connectivity in the human brain. PLoS Comput Biol. 2010; 6:e1000808.

21. Beucke JC, Sepulcre J, Talukdar T, Linnman C, Zschenderlein K, Endrass T, Kaufmann C, Kathmann N. Abnormally high degree connectivity of the orbitofrontal cortex in obsessive-compulsive disorder. JAMA Psychiatry. 2013; 70:619-629.

22. Stufflebeam SM, Liu H, Sepulcre J, Tanaka N, Buckner RL, Madsen JR. Localization of focal epileptic discharges using functional connectivity magnetic resonance imaging. J Neurosurg. 2011; 114:1693-1697.

23. Wang X, Xia M, Lai Y, Dai Z, Cao Q, Cheng Z, Han X, Yang L, Yuan Y, Zhang Y, Li K, Ma H, Shi C, et al. Disrupted resting-state functional connectivity in minimally treated chronic schizophrenia. Schizophr Res. 2014; 156:150-156.

24. Delbeuck X, Van der Linden M, Collette F. Alzheimer's disease as a disconnection syndrome. Neuropsychol Rev. 2003; 13:79-92.

25. Smith SM, Miller KL, Salimi-Khorshidi G, Webster M, Beckmann CF, Nichols TE, Ramsey JD, Woolrich MW. Network modelling methods for FMRI. Neuroimage. 2011; 54:875-891.

26. Achard S, Salvador R, Whitcher B, Suckling J, Bullmore E. A resilient, low-frequency, small-world human brain functional network with highly connected association cortical hubs. J Neurosci. 2006; 26:63-72.

27. Tomasi D, Volkow ND. Functional connectivity density mapping. Proc Natl Acad Sci USA. 2010; 107:9885-90.

28. Wang L, Dai Z, Peng H, Tan L, Ding Y, He Z, Zhang Y, Xia M, Li Z, Li W, Cai Y, Lu S, Liao M, et al. Overlapping and segregated resting-state functional connectivity in patients with major depressive disorder with and without childhood neglect. Hum Brain Mapp. 2014; 35:1154-66.

29. Yeo BT, Krienen FM, Sepulcre J, Sabuncu MR, Lashkari D, Hollinshead M, Roffman JL, Smoller JW, Zöllei L, Polimeni JR, Fischl B, Liu H, Buckner RL. The organization of the human cerebral cortex estimated by intrinsic functional connectivity. J Neurophysiol. 2011; 106:1125-1165.

30. Yang M, Chen HJ, Liu B, Huang ZC, Feng Y, Li J, Chen JY, Zhang LL, Ji H, Feng X, Zhu X, Teng GJ. Brain structural 
and functional alterations in patients with unilateral hearing loss. Hear Res. 2014; 316:37-43.

31. Miller EK, Cohen JD. An integrative theory of prefrontal cortex function. Annu Rev Neurosci. 2001; 24:167-202.

32. Ray RD, Zald DH. Anatomical insights into the interaction of emotion and cognition in the prefrontal cortex. Neurosci Biobehav Rev. 2012; 36:479-501.

33. Lévesque J, Eugène F, Joanette $Y$, Paquette V, Mensour B, Beaudoin G, Leroux JM, Bourgouin P, Beauregard M. Neural circuitry underlying voluntary suppression of sadness. Biol Psychiatry. 2003; 53:502-510.

34. Phan KL, Fitzgerald DA, Nathan PJ, Moore GJ, Uhde TW, Tancer ME. Neural substrates for voluntary suppression of negative affect: a functional magnetic resonance imaging study. Biol Psychiatry. 2005; 57:210-219.

35. Phillips ML, Drevets WC, Rauch SL, Lane R. Neurobiology of emotion perception I: The neural basis of normal emotion perception. Biol Psychiatry. 2003; 54:504-514.

36. Venkatraman V, Rosati AG, Taren AA, Huettel SA. Resolving response, decision, and strategic control: evidence for a functional topography in dorsomedial prefrontal cortex. J Neurosci. 2009; 29:13158-13164.

37. Kerns JG, Cohen JD, MacDonald AW, Cho RY, Stenger VA, Carter CS. Anterior cingulate conflict monitoring and adjustments in control. Science. 2004; 303:1023-1026.

38. Raichle ME, MacLeod AM, Snyder AZ, Powers WJ, Gusnard DA, Shulman GL. A default mode of brain function. Proc Natl Acad Sci USA. 2001; 98:676-682.

39. Gusnard DA, Akbudak E, Shulman GL, Raichle ME. Medial prefrontal cortex and self-referential mental activity: relation to a default mode of brain function. Proc Natl Acad Sci USA. 2001; 98:4259-4264.

40. Wheeler ME, Shulman GL, Buckner RL, Miezin FM, Velanova K, Petersen SE. Evidence for separate perceptual reactivation and search processes during remembering. Cereb Cortex. 2006; 16:949-959.

41. Leech R, Kamourieh S, Beckmann CF, Sharp DJ. Fractionating the default mode network: distinct contributions of the ventral and dorsal posterior cingulate cortex to cognitive control. J Neurosci. 2011; 31:3217-3224.

42. Broyd SJ, Demanuele C, Debener S, Helps SK, James CJ, Sonuga-Barke EJ. Default-mode brain dysfunction in mental disorders: a systematic review. Neurosci Biobehav Rev. 2009; 33:279-296.

43. Dai Z, Yan C, Li K, Wang Z, Wang J, Cao M, Lin Q, Shu N, Xia M, Bi Y, He Y. Identifying and Mapping Connectivity Patterns of Brain Network Hubs in Alzheimer's Disease. Cereb Cortex. 2015; 25:3723-42.

44. Hawellek DJ, Hipp JF, Lewis CM, Corbetta M, Engel AK. Increased functional connectivity indicates the severity of cognitive impairment in multiple sclerosis. Proc Natl Acad Sci USA. 2011; 108:19066-19071.

45. Laugen HPO, Brännström J, Aarstad HJ, Vassbotn FS, Specht K. Functional-structural reorganisation of the neuronal network for auditory perception in subjects with unilateral hearing loss: Review of neuroimaging studies. Hear Res. 2016; 332:73-79.

46. Schmithorst VJ, Plante E, Holland S. Unilateral deafness in children affects development of multi-modal modulation and default mode networks. Front Hum Neurosci. 2014; $8: 164$.

47. Falchier A, Clavagnier S, Barone P, Kennedy H. Anatomical evidence of multimodal integration in primate striate cortex. J Neurosci. 2002; 22:5749-5759.

48. Rockland KS, Ojima H. Multisensory convergence in calcarine visual areas in macaque monkey. Int $\mathrm{J}$ Psychophysiol. 2003; 50:19-26.

49. Bavelier D, Dye MW, Hauser PC. Do deaf individuals see better. Trends Cogn Sci. 2006; 10:512-518.

50. Sladen DP, Tharpe AM, Ashmead DH, Wesley GD, Chun MM. Visual attention in deaf and normal hearing adults: effects of stimulus compatibility. J Speech Lang Hear Res. 2005; 48:1529-1537.

51. Li Y, Booth JR, Peng D, Zang Y, Li J, Yan C, Ding G. Altered intra- and inter-regional synchronization of superior temporal cortex in deaf people. Cereb Cortex. 2013; 23:1988-1996.

52. Liang X, Zou Q, He Y, Yang Y. Coupling of functional connectivity and regional cerebral blood flow reveals a physiological basis for network hubs of the human brain. Proc Natl Acad Sci USA. 2013; 110:1929-1934.

53. Bullmore E, Sporns O. Complex brain networks: graph theoretical analysis of structural and functional systems. Nat Rev Neurosci. 2009; 10:186-198.

54. Myers-Schulz B, Koenigs M. Functional anatomy of ventromedial prefrontal cortex: implications for mood and anxiety disorders. Mol Psychiatry. 2012; 17:132-141.

55. Rauch SL, Shin LM, Phelps EA. Neurocircuitry models of posttraumatic stress disorder and extinction: human neuroimaging research--past, present, and future. Biol Psychiatry. 2006; 60:376-382.

56. Langers DR, van Dijk P. Robustness of intrinsic connectivity networks in the human brain to the presence of acoustic scanner noise. Neuroimage. 2011; 55:1617-1632.

57. Folstein MF, Folstein SE, McHugh PR. "Mini-mental state". A practical method for grading the cognitive state of patients for the clinician. J Psychiatr Res. 1975; 12:189-198.

58. Chao-Gan Y, Yu-Feng Z. DPARSF: A MATLAB Toolbox for "Pipeline" Data Analysis of Resting-State fMRI. Front Syst Neurosci. 2010; 4:13.

59. Ashburner J, Friston KJ. Unified segmentation. Neuroimage. 2005; 26:839-851.

60. Liao XH, Xia MR, Xu T, Dai ZJ, Cao XY, Niu HJ, Zuo XN, Zang YF, He Y. Functional brain hubs and their test-retest reliability: a multiband resting-state functional MRI study. Neuroimage. 2013; 83:969-982.

61. Zuo XN, Ehmke R, Mennes M, Imperati D, Castellanos FX, Sporns O, Milham MP. Network centrality in the human functional connectome. Cereb Cortex. 2012; 22:1862-1875. 
62. Tomasi D, Wang GJ, Volkow ND. Energetic cost of brain functional connectivity. Proc Natl Acad Sci USA. 2013; 110:13642-13647.

63. Nichols TE, Holmes AP. Nonparametric permutation tests for functional neuroimaging: a primer with examples. Hum Brain Mapp. 2002; 15:1-25.

64. Eklund A, Nichols TE, Knutsson H. Cluster failure: Why fMRI inferences for spatial extent have inflated falsepositive rates. Proc Natl Acad Sci USA. 2016; 113:7900-5.

65. Power JD, Barnes KA, Snyder AZ, Schlaggar BL, Petersen SE. Spurious but systematic correlations in functional connectivity MRI networks arise from subject motion. Neuroimage. 2012; 59:2142-54.

66. Welvaert M, Rosseel Y. On the definition of signal-to-noise ratio and contrast-to-noise ratio for FMRI data. PLoS One. 2013; 8:e77089. 